\title{
DIAGNOSIS AND TREATMENT OF ECZEMA
}

\author{
By J. E. M. WIGLEY, M.B., B.S., F.R.C.P. \\ (Physician, St. John's Hospital, Charing Cross Hospital, etc.)
}

Whilst an entirely satisfactory definition of eczema is difficult to find, the following working one seems to me a fair practical guide. Eczema is an inflammatory reaction of the skin, shown by various combinations of the following lesionserythema, papules, vesicles, crusts and scales; accompanied by varying degrees of itching, a considerable resistance to treatment and a marked tendency to relapse.

Eczema is not, strictly speaking, a disease, but rather an altered reaction of the skin to a variety of provocative agents, both external and internal. In 1906 von Pirquet suggested the word "allergy" to designate "an altered capacity to react." From this it would appear that the adjective "allergic" is only strictly applicable to tissues which have this "altered capacity" and that whilst patients or parts of patients may be "allergic" it is quite incorrect to speak of diseases as allergic. However, I fear that the latter employment of the word has almost become sanctioned by use (or rather misuse) and that eczema is a condition to which it is very frequently applied. As the condition called eczema is essentially a manifestation of the altered capacity to react, to speak of allergic eczema, in addition to being wrong, is an unnecessary use of words and one might have thought the doubtfully desirable attempts at brevity, "streamlining," as it is often miscalled, would have made it anathema to the modern speaker or writer.

The question frequently arises as to what is the difference between dermatitis and eczema and to which cases should either term be applied. The answer is that fundamentally there is no difference. As Bruno Bloch (I929) stated "one can produce in them (i.e. normal persons sensitised to the primrose) every clinical, macroscopic and microscopic histologic change which is considered characteristic of true eczema." The common phenomena which are seen in the localised lesion called "irritant dermatitis" (Sequeira) or "contact dermatitis" are redness, vesication, exudation and the formation of scales and crusts. These are exactly the same as those seen in any area of the more widely distributed eruption called eczema and much histological study has failed to reveal any real difference in the two conditions. It is an exceedingly well known observation that the patient who begins his skin troubles with a localised eruption on one part (resulting from contact with some "irritant" in his occupation) may develop a widespread and symmetrical eruption which con- forms to all the criteria of eczema. This eruption will persist and relapse in spite of all the best efforts to avoid repetition of the "occupational irritant" contacts in exactly the same way as the condition called "constitutional" eczema. That there must be some physiological cause for this underlying change in the reaction of the skin (allergy) is $\vec{\circ}$ obvious, and many and varied have been the investigations undertaken to find it: So far all these investigations have failed to show the existence of any metabolic disturbance which is common to all or even to many types of eczema and the mysterious change which we call "hypersensi- N tivity" remains a mystery. So far as I can find, i cutaneous hypersensitivity has not been shown to $\vec{\sigma}$ exist at birth, but it is evident that it may be $\vec{\circ}$ acquired at a very early age. I am inclined to $\omega$ think that it is always originally a contact with some external provocative agent. I am in agreement with Ingram (I933) when he writes that o "that external stimulus to eczema can be anything under the sun, including the sun." That $\vec{\oplus}$ cutaneous hypersensitivity can be quite local is a accepted fact and it is equally important to re member that the skin can become increasing sensitive to provocative agents so that, eventually, the slightest exposure is capable of producing the eruption.

Unfortunately the word dermatitis has become very largely associated with the Workman's Compensation Act where the compensatable condition is called "Dermatitis produced by dust or liquids" and it is a common experience that a sharp legal distinction is drawn between this condition and "constitutional eczema." I think that, as a practical procedure, it is necessary to bow to the authority of the law and reserve the word dermatitis for those cases in which the external provocative agent can be demonstrated and the contact with such an agent can be shown to have taken place in the course of the patient's employment. This will frequently avoid disappointment and possible financial loss to patients in whose minds $N$ the words dermatitis and compensation are almost $N$ synonymous.

I want to make it quite clear that I do not intend my remarks to apply to certain types of eczema, both infantile and otherwise, in which there is definite association with asthma, hay fever, $\mathbb{D}$ and other allied conditions. These are being dealt : with in another article.* What I have attempted *In a future issue. $-E d$. 
to emphasise is that the eruption, which is evidence of the altered reaction of the hypersensitive skin, is one and the same whether it be called eczema or dermatitis, with one or more qualifying adjectives. There is much to be said for the compromise term of eczematous dermatitis.

\section{Clinical Appearances}

Eczema is one of the most multiform of skin diseases. The cardinal features consist of erythema superficial oedema, and an inflammatory cellular infiltration which are responsible for papules, vesicles, and exudation, crusts and scales. They are not usually all present in the same lesion or at the one time but there is practically always more than one of them. The individual lesions may be quite sharply demarcated in their earlier stages but there is a marked tendency towards blurring of outlines as the eruption spreads and relapses. The colour varies from the brilliant red of the acute lesion, through shades of pink and mauve to a distinctive blue colour when the dependent parts are affected. Scratch marks, both linear and "papular," often blood crusted, are almost invariably to be seen. In long-standing cases there are usually cracks and fissures along lines of flexion and the skin may become tough and thickened, giving an appearance which has been likened to "shagreen leather."

Although there is practically always a multiformity of primary lesions, there is usually a predominance of one lesion, and for purposes of description it is convenient to describe various phases of eczema according to the predominating lesion.

(I) Erythematous Eczema.-This may be regarded as the primary phase and is usually the more acute one. It is most characteristically seen about the face and hands though it may occur elsewhere. Bright red, hot and tense blotches appear which may coalesce into ill-defined patches. They may be markedly symmetrical and difficult to distinguish from erysipelas. There is seldom any constitutional disturbance, e.g. rise of temperature, in eczema and the erythema is usually broken up by areas of normal skin. The outlines of the erythema are usually indefinite and the colour fades gradually into the surrounding skin. This is in sharp contrast to erysipelas. Careful study of the surface will usually show some tiny vesicles and as the condition subsides there is some scaling.

(2) Papular Eczema.-This phase is chiefly re-markable for aggregations of or areas covered with, papules. The papules are rounded or acuminate, about the size of pins' heads, and are usually situated on an erythematous base. Occasionally they appear without any previous erythema, forming a chronic type of eczema generally associated with intolerable itching. The papules vary in colour, being pinkish red on the trunk and purplish on the limbs. Examination with a hand lens often reveals a minute vesicle surmounting some of the papules. Evidence of scratching, e.g. blood crusted papules, is nearly always to be seen. In this phase eczema may be arranged in small irregular groups, when it is to be distinguished from secondary syphilis, situated anywhere on the skin, showing some predilection for the extensor aspects of the arms and thighs. At times, when the papules have persisted somewhat, the rubbing and scratching may transform them into flat shiny lesions which may be difficult to distinguish from lichen planus. The intense irritation of this phase of eczema may cause such violent scratching and laceration that pyogenic infection easily takes place and the area may become covered with purulent scabs.

(3) Vesicular Eczema.-Here the intense oedema causes the formation of superficial vesicles, "water blisters," nearly always on an erythematous base. It is this appearance which originally suggested the name of eczema (from $\dot{\varepsilon} \kappa \gamma \varepsilon \omega=$ to boil over). The vesicles are easily ruptured, either by friction or "vis-a-tergo," and a clear yellowish serous fluid exudes which coagulates to form sticky yellowish crusts and has the property of stiffening linen. When this "weeping" is a marked feature the name of eczema madidans is sometimes used. The vesicular phase is seen most often where the skin is delicate, as about the flexures of the joints, forearms, and scrotum, but may occur anywhere, even on the scalp.

(4) Lichenified Eczema.-It is not uncommon that patches or areas of eczema, which have begun as one of the above phases, settle down into a chronic, very itchy and thickened phase. In this phase the skin is usually of a brownish, rather "dirty" colour and feels much thicker than normal when pinched up between the fingers. The surface has a shiny, crosshatched appearance which has been aptly likened to shagreen leather, whilst around the area a varying number of flat rounded papules gradually fade into the surrounding skin. This lichenification, as it is called, is the result of oft-repeated friction, usually by rubbing and scratching. The latter usually leaves denudation of the surface epithelium which can easily be recognised.

Various qualifying names are often applied to eczema. These are really only emphasis of the predominating phase in individual cases, but may be useful for description. For example, "weeping 
eczema" denotes that exudation is the most marked feature; "squamous eczema," harsh, scaling and extensive desquamation; "fissured eczema," superficial or deep fissures; "crackled eczema," irregular superficial cracks in the epidermis giving an appearance suggesting crackled china, etc. Pustular eczema is a term used when there is much secondary pus-coccal infection, which is also responsible for many of the "warty" lesions of "papillomatous eczema."

In making the diagnosis of eczema, the multiformity of the lesions must always be remembered and I think that to add to the definition given in the opening paragraph would be an unnecessary use of words (which I have already deplored). A few words about some of the conditions which are more commonly mistaken for eczema would not be out of place.

(I) Erythema Multiforme.-This is essentially a symmetrical eruption, most commonly affecting the forearms and backs of the hands and/or the shins and dorsa of the feet. The lesions are erythematous, have a smooth "velvety" feeling, and often a ringed or target-like appearance.

(2) Exfoliative Dermatitis.-This is usually a universal, dry eruption with much scaling but little infiltration. The multiformity of lesions of eczema is in sharp contrast and it is extremely rare that eczema does not spare some areas of the skin.

(3) Lichen Planus.-This eruption always shows the smooth, flat-topped burnished papules of a characteristic mauve or violaceous colour. Lesions in the mouth, on the glans penis or about the vulva are often present. Vesiculation is rare and "weeping" almost unknown and the brown to blackish pigmentation left by fading lesions is more than suggestive. On occasion, papular eczema may be difficult to distinguish from lichen planus, but a careful study of every part of the eruption will usually settle the diagnosis.

(4) Intertrigo.-This is a word used to describe moist, red, hyperaemic areas which occur about skin surfaces which are in opposition, e.g. the submammary regions, the axillae, the folds in fat babies and the chins which are often called double. The surface is usually raw and secretes a watery fluid which does not coagulate like the exudation of eczema.

(5) Paget's Disease of the Nipple.-This very rare affection is characterised by being more sharply demarcated than eczema affecting the same area. The nipple is usually retracted and the area feels indurated, whilst the course is more steadily pro- gressive without the remissions and relapses so usual in eczema. If there is real doubt a biopsy should always be done, as Paget's disease is car- $\frac{\mathbb{Q}}{\mathbb{Q}}$ cinomatous "ab initio."

(6) Psoriasis.-This eruption, with its dry, $\underset{\vec{F}}{\vec{S}}$ silvery (or mica-like) scales on a salmon-pinkish 0 coloured, demarcated erythema and its very characteristic distribution should seldom present $\frac{}{\sigma}$. difficulties. Dry scaling and partly lichenified $\stackrel{\varnothing}{\varrho}$ areas of chronic eczema may resemble psoriasis, which eruption may become moist and even कs weeping if it is much scratched. However, careful search will usually reveal at least one typical lesion.

(7) Pityriasis Rosea.-The typical lozenge-shaped pink macule, with its cafe-au-lait centre and its 3 collarette scaling (attached about the periphery iN and free towards the centre) is distinctive. The i previous "herald patch" and "old-fashioned bathing suit" distribution will usually make the diagnosis clear.

(8) Sycosis of the Beard.-This is characteristi- cally a pustular folliculitis and only in chronic cases $?$ may present difficulty. Even then the follicular affection is generally evident, whilst the limitation $\vec{v}$ to hairy regions, the slight amount of irritation ang the absence of weeping should decide the matter

(9) Syphilis.-It is only in the papular and scaly forms that confusion should arise. The absence of irritation, symmetrical distribution, uniformity and induration of lesion, brownish colour and enlargement of lymphatic glands should point to the diagnosis. The result of the Wassermann reaction and the response to antisyphilitic treatment will decide.

(Io) Seborrhoeic Dermatitis.-This eruption usually has a predilection for the front of the chest, the interscapular region, the scalp and behind the ears. The scales usually have a yellowish greasy appearance, with often a circinate arrangement. However the picture may be very confused by an eczematous reaction being provoked in a "seborrhoeic" subject, by scratching or injudicious treatment and in many cases there is present a compound affection to which the name of seborrhoeic eczema is applicable.

(II) Scabies.-In any patient suffering from an itching eruption, scabies should be excluded before 0 arriving at any other diagnosis. Many cases of $\mathbb{D}$ scabies show eczematous lesions, usually the result of inapt treatment or scratching and the picture may be confused. The very characteristic distribution of scabies, as well as the finding of burrows 
and, for preference, of the acarus will decide the question finally.

Having made the diagnosis of eczema one has really only given a label to the clinical picture, and it is vital that every attempt should be made to discover the underlying cause of the eruption if rational, and possibly successful treatment is to be carried out. As I have pointed out, the eruption which we call eczema is the outward and visible sign of the inward and allergic reaction of the patient's skin. What is the provocative agent which has set off that altered reaction in the particular case under consideration, in other words, what is the "detonator?" Broadly speaking the "detonators" may be placed in two large groups:-

I. Those which are external to the body and act by actual contact with, or reasonably close proximity to the skin.

2. Those which are within the body and act most probably through the blood stream. These substances may be ingested or inhaled, or may be products of disordered metabolism.

For very many years, even centuries, dermatologists and others considered the latter as the more important group and dyscrasias or diatheses were largely invoked as causes of eczema. In more recent and pseudo scientific language, "lack of calcium," "lack of vitamins," "too much acid," "autointoxication," "worry" and, of course, "nerves," have carried on these vague theoretical generalisations. None of these factors has been consistently proved to exist in any series of cases, and they have continued chiefly as an apparently impressive cloak for ignorance; satisfying the "little learning" which the quasi-scientifically halfinformed public love to think it possesses.

That, in some cases of eczema, the provocative agent is of internal origin cannot be denied. The example which immediately springs to the mind is the eczematous eruption which may appear at any time during the course of injection of the N.A.B. compounds, gold salts, etc., but even here it may be difficult to quite exclude an external detonator. In the words of Bruno Bloch2 (r929): "The more pains one takes in the effort to find the cause in cases of eczema as regards both the history and the functional tests, the more exogenous factors will be found as the real basis for this disease." This view has been shown to be correct by practically all writers and workers since then and there can be no doubt that eczema should not be considered as due to an internal cause until every attempt has been made to exclude external provocative agents ("irritants") as the principal factors.

How is this to be done? It may be quite easy, as in cases where the eczema follows the use of hair dyes; strong "cleansing agents"; wearing of irritant clothing or ornaments; using unsuitable remedies or handling certain plants such as the primrose or chrysanthemum, when it appears first on the part of the skin exposed. On the other hand, it may be extremely difficult, especially when one remembers the universal distribution of possible irritants and the almost unbelievably small quantities which may produce even the most severe reactions. Here we can observe the tremendous variation in susceptibility to sensitisation on the part of different individuals, as well as the mystery of allergic "memory." The original sensitising "contact" may have occurred in the nearly forgotten past.

The first approach is by way of the history. "The proper taking and evaluation of the history may be of greater practical help than are all the other investigative measures combined" (Sulzberger,5 Dermat. Allergy, I940, p. I00). This is, of necessity, arduous and time-consuming and may call for ability both in detection and cross-examina- $\infty$ tion which would have done credit to Sherlock 을 Holmes or Lord Birkenhead. In the taking of such a history it is of the utmost importance not to be influenced by the effect of substances to which custom has attributed, often erroneously, the capa $\overrightarrow{-} \vec{\bullet}$ bility of producing eczema. Try and judge everg event by the existence of facts and not be influs enced by what "should" have occurred. Not the least difficulty will be that of convincing the patien t' of the necessity for suspecting even the seemingly most innocuous substances. Eczema has for so long been regarded as of internal origin that many patients will be surprised, nay, even offended, if it is suggested that the "gouty" eczema is due to wearing dyed gloves or sock suspenders. The time and energy spent will not be grudged when it is realised that this is the dermatosis in which the prospect of finding the cause is greatest and in which the practical results obtained are the most gratifying.

It is not possible, in this paper, to go into any great detail about the form this history taking should follow, but some general indications may serve as a guide in the search for the suspected provocative agent:-

I. The site of the first appearance or the site of predilection of the eruption.-The face (eyelids) and scalp are first affected by hair dyes, cosmetics, nail $\mathrm{\omega}$ varnishes, hatbands; the neck and face (eyelids) by furs, fur dyes, collars, scarves, flowers, etc.; the hands and forearms by occupational substances, $\mathbb{D}$ soaps, cleansers, gloves, etc.; the torso by silk, wool, other materials, dress shields, corselet-belts (girdles), substances used in massaging, bath salts, etc.; the thighs by suspenders, etc.; the feet and 
legs by shoe leather, shoe polishes, sock materials, suspenders, etc.; the spectacle areas by metal or artificial tortoise-shell, and so forth.

Oedema of the eyelids, especially in conjunction with eczema anywhere about the head and neck, is always very suggestive of an external irritant and usually one of some degree of volatility.

II. The occupation of the patient.-In assessing this factor it is necessary to know or to become acquainted with the multitudinous substances which represent the hazards of different occupations. It is quite impossible to give even an approximate list of these but they may conveniently be divided into two groups:-

(a) Primary irritants, which cause an eczematous eruption on the skin of the vast majority of all people handling them. Examples are carbon tetrachloride and poison ivy.

(b) "Sensitising" irritants, in which repeated contact is usually necessary to produce the eczema. Examples are alkalis, cement, plastics, etc.

In assessing the "occupational" factor in any case it is important to remember that the alkaline soaps, degreasers, and other cleansing agents used to remove visible evidence of toil are often as much a factor in producing the eruption as the noxious agents which are far more commonly blamed.

III. The hobbies and habits of the patient.-In the amateur photographer the various chemicals are suspect; in the horseman, leather, leather polishes, saddle soap, etc.; in the violinist rosin, chin-rest, wood or wood finishes; in the mah jong player the lacquered tiles; in the golfer, plants and grasses, leather, club polishes, etc. In people who keep domestic animals substances employed in their cleanliness, e.g. lime wash for fowl-houses. Contraceptives and douches must be remembered in cases where the eczema is primarily situated about either male or female genitalia.

Cosmetics, whether powders, hair dyes, nail varnishes or their removers, mascara, perfumes, deodorants, depilatories, antihydrotics, face creams, wrinkle removers, hair lotions, setting lotion, etc. etc., may all have to be considered. Children's toys and playgrounds may provide the clue, as well as the various furnishings and decorations of rooms and the substances used in their cleaning, etc.

In all these it is necessary to consider not only the substances used by the patient but also those used by friends, playmates and intimates.
IV. All remedies used in previous treatment.This is most important and may well include sub- $\frac{a}{3}$ stances which you yourself have ordered as well as $\mathbb{0}$ those advocated by advertisement or recom- $c$. mended by friends. In this group all sulphonamide applications are particularly suspect, as are local ${ }^{\circ}$ anaesthetics of the Percain and Benzocain type, as well as Antipeol. These are especially potentialo sensitisers. At the same time it must never be $\frac{\bar{c}}{\frac{1}{?}}$ forgotten that even the most universally soothing $\stackrel{\widetilde{\alpha}}{\widetilde{\alpha}}$ applications may irritate or sensitise some patient's® skin.

Patch-testing. - The careful taking of the history, $\stackrel{\overrightarrow{\vec{\omega}}}{\vec{\omega}}$ which may need to be repeated many times, will $\stackrel{\omega}{\circ}$ frequently be found to have established the identity of the causal agent, when further testing $\underline{3}$. will be unnecessary. On the other hand, this history taking may only have narrowed the ground ori have made so many substances suspect that skin $\hat{\circ}$ tests must be undertaken in order to support or $\overrightarrow{0}$ complement the history and the clinical findings. $\mathrm{O}$ Haphazard skin testing may well incriminate so의 many substances, including many that the patient cannot reasonably have come into contact with, $\stackrel{5}{8}$ that the resulting confusion may be more bewildering than the original problem. There must $\overrightarrow{0}$ always be a history of opportunity for exposure gof the suspected substance, though the fact that $t^{\circ}$ may be an old history ("allergic memory") will cạt을 for considerable skill in eliciting it.

The "Patch Test" (or eczema test) is to be distinguished from the scratch or intradermal test. Essentially, it consists of applying the suspected $\stackrel{\mathcal{O}}{\overrightarrow{2}}$ substance to the undamaged skin and eliciting an $\overrightarrow{\overrightarrow{0}}$ epidermal response in the form of an eczematous 3 patch. The scratch test is done by inserting the substance into the skin and eliciting a vascular response, usually in the form of a wheal. Of응 course the best skin test is a repetition of the original contact with the suspected substance, 3 . under the same conditions, but this is, obviously, frequently not practicable.

Before describing the test it should be made quite clear that all testing is to be avoided during ? the active phase of an acute eruption. In addition? to the very real risk of making the whole eruption worse and more intractable, the results obtained N may be quite misleading. "Multiple sensitivity" is more likely during the active phase of an eruption and, at times, one may find the skin in a negative phase.

Another warning is that the test should not beo applied to a site where any ensuing cosmetic defect产 may be extremely disagreeable or disastrous to the ${ }^{+}$ patient. The face should obviously be avoided, as $\frac{T}{0}$ well as decollete areas in women, though this may leave little space in certain professions. Always? 
obtain the patient's consent to any testing, after fully explaining the process to them.

To carry out the test:-

A small piece of the suspected substance, or a piece of white linen soaked in the solution or covered with the grease or with the water-moistened powder or other material is applied to the unbroken, hairless skin. If the material can be cut into a distinctive shape it is an advantage but the overall size should not be larger than a postage stamp. The material is covered with a somewhat larger piece of impermeable material, e.g. cellophane, used photographic film, or oiled silk. The whole is kept in place by adhesive strapping or a bandage if the patient is known to react unduly to strapping. A similar "control" application is made, without the suspect substance, of course. It is usually inadvisable to apply more than half a dozen tests to one patient at a time. The patches are left in place for 24 to 48 hours, though the patient must be instructed to remove any or all of them at once should the irritation become intolerable. Of course, the patches are carefully marked. When the patches are removed, the sites are thoroughly cleansed and the reactions noted. A positive reaction must be more than an erythema, the papules, vesicles, or other characteristics of eczema being essential. It is also important to be certain that the reaction has not been caused by the use of a "primary irritant" in too strong a concentration. Attention to detail and observation of the reaction for a few days will settle this.

If there is no evident reaction at the site when it is first seen, it is necessary to see it at short intervals afterwards, as "delayed reactions" occur not infrequently. Delay up to as many as 30 days has been reported.

\section{Evaluation of the Patch Test}

A positive patch test suggests, though it does not prove, that the substance used is the cause of the presenting eczema. If this supports the history, part of which must be that there was an opportunity of contact about the time of the onset of the eruption, the suggestion is made a reasonable certainty.

A negative patch test, after the necessary "delay" even, is very much less conclusive. There are many variations in the degree of sensitivity and only local sensitivity is well known. The conditions of contact may not be adequately reproduced by the testing process.

To sum up:- The diagnosis of eczema is made from the consideration of the following factors:-
I. The clinical appearances of the eruption and its accompanying symptoms.

II. The history, in particular that part bearing on contact with potential irritants.

III. The relapsing nature of the eruption.

IV. The response to treatment.

\section{Treatment}

The treatment, or perhaps better, the management of eczema is such a comprehensive subject that it is quite impossible to do more than indicate some general lines and to give some warnings in this paper. I think it is worth the risk of being accused of reiteration to stress the importance of a very thorough search for external irritant causes in every case, as it is obvious that the most expertly applied remedies will fail if the exciting irritant is still having even occasional contact with the patient.

A very important thing to remember is that the proper method of application of a remedy is fully as important as the choice of a suitable remedy. Time is well spent in giving definite and detailed instructions to a patient as to how to use whatever is prescribed and many more pleasing "cures" will result than if he or she is given some lotion or ointment and just told to "go home and use it." It is also very much better to know how and when to use a few comparatively simple remedies than to try the rather haphazard use of many. Simple prescriptions, containing few ingredients, are better than the possibly more imposing seeming "shot gun" ones. In addition to increasing the experience with a few drugs, this also minimises the likelihood of prescribing a drug to which the patient already is, or will become, sensitised.

"Primum non nocere" is a very first law in the treatment of eczema, as much as in any branch of medicine. The patient's symptoms are of the greatest help in guiding the use of applications. Generally speaking, any application which the patient finds soothing or comforting is likely to be helpful, whilst any application which the patient finds irritating is likely to retard the improvement, however perfect the remedy "should" be for the condition-in theory.

A great deal of the success in the treatment of eczema depends on the ability to relieve or control irritation. The rubbing and scratching this very trying symptom evokes often aggravates the eczematous process, as well as the irritation, and so a vicious circle is easily established. Here it is very necessary to give a warning against the use of the local anaesthetic drugs as local applications, 


\section{DIAGNOSIS AND TREATMENT OF ECZEMA}

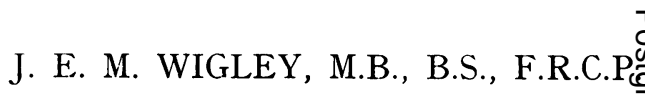

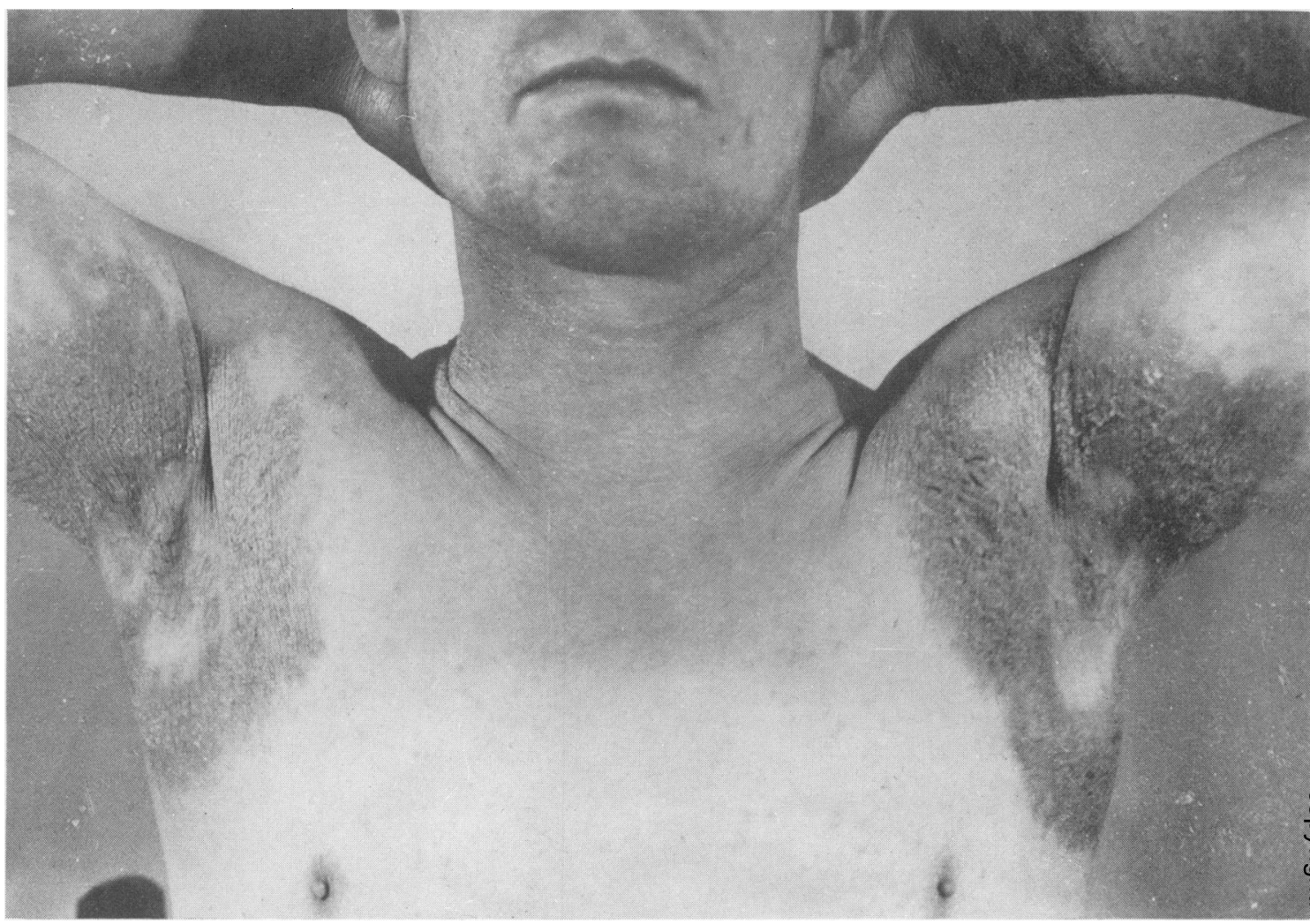

FIG. I.-Eczematous eruption from contact with dyed clothing (shirt). (From the collection of photographs at

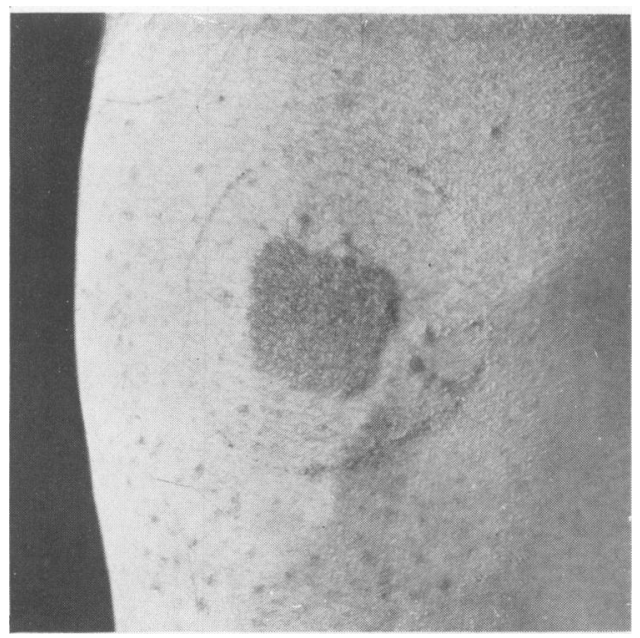

\section{St. John's Hospital for Diseases of the Skin, Lisle Streel, Leicester Square.)}

Fig. 2.-Positive patch test. (From the collection of photographs at St. John's Hospital for Diseases of the Skin, Lisle Street, Leicester Square.) 


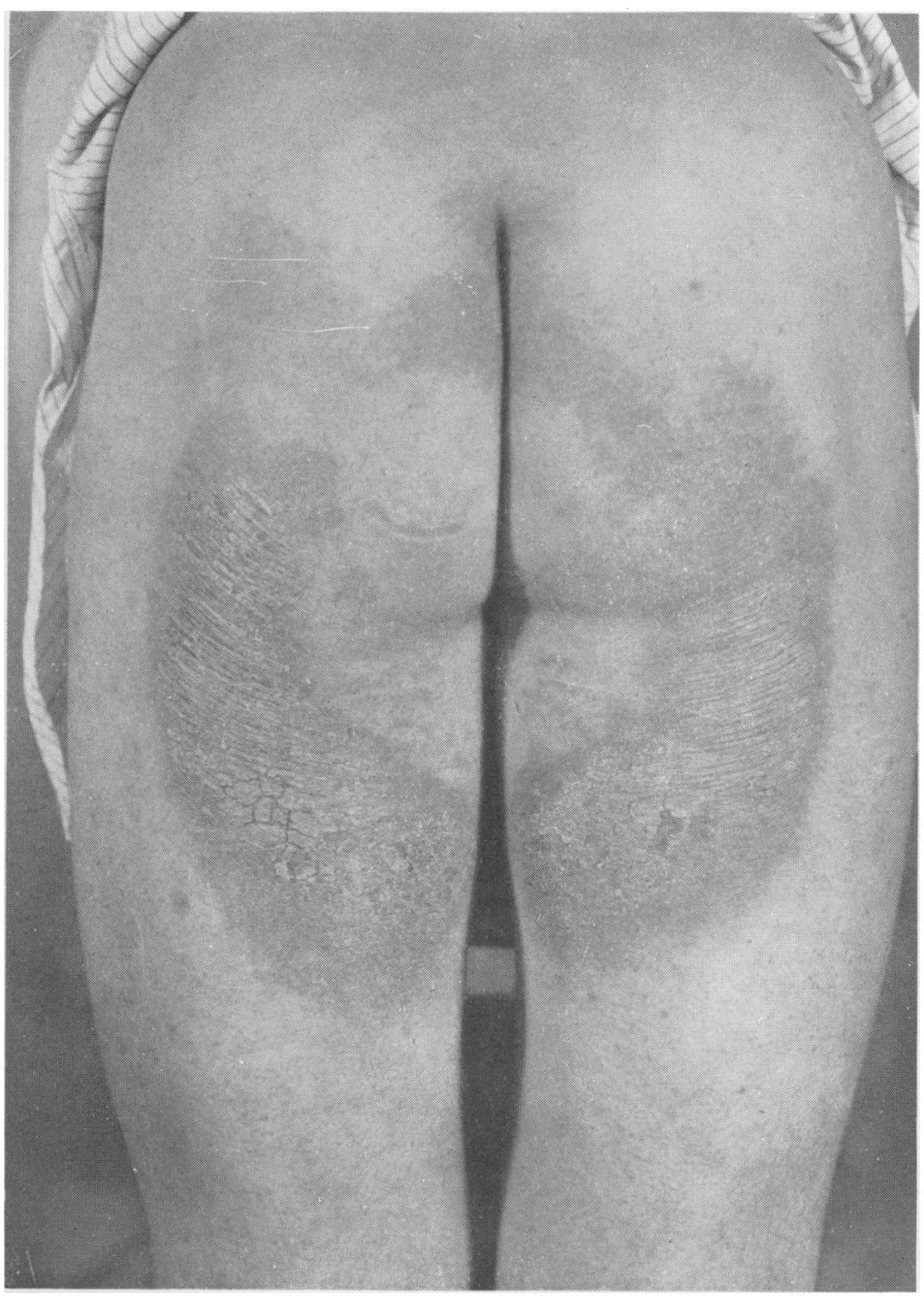

FIG. 3.-Eczematous eruption from contact with polished lavatory seat. (From the collection of photographs at St. John's Hospital for Diseases of the Skin, Lisle Street, Leicester Square.)

FIG. 4--Positive patch test with superimposed blister from a primary irritant. (Both these photographs are from the collection at St. John's Hospital for Diseases of the Skin, Lisle Street, Leicester Square.)

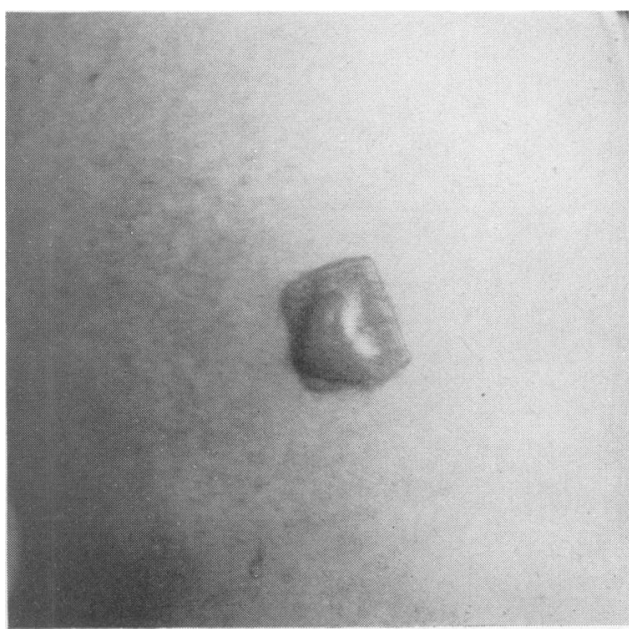


such as benzocaine, percainal, etc. Often, even if they do appear to relieve the irritation at first, when the effect wears off the irritation returns with increased fury. More serious than this, these drugs are frequently skin sensitisers; they quite often produce or aggravate an eczematous eruption. Usually it is necessary to control irritation by the use of general sedatives. Barbiturates and salicylates are usually the most effective, and a much better result will nearly always be obtained if these drugs are given in divided doses, spread over the whole twenty-four hours rather than in one larger dose at bedtime. Other drugs such as the bromides, chloral hydrate, may be used but at times it may test the skill of the most experienced physician to get the patient any relief. In general, opium and its derivatives must be avoided. These drugs often have a tendency to aggravate, or even to cause, itching and there is always the fear of establishing the habit. The value of rest, both local and general, cannot be exaggerated.

\section{Local or External Treatment}

A most useful appliance in controlling irritation is the ordinary "wooden splint." A suitably padded and well applied splint will frequently prove so successful that the patient will insist on its application to prevent the nocturnal, or even diurnal "subconscious" scratching. So what may appear at first to be an inhumane procedure will be found to be a great boon, and this applies to adults as well as children.

Some general indications of local remedies for the principal phases of eczema, as set out above, will probably be helpful.

In the eczematous phase an absorbent, astringent and sedative application is indicated. This is best provided in the form of a lotion or powder and ointments are contraindicated. The renowned Calamine lotion combines the effects of both the evaporation of the lotion and the deposition of the powder and, as all lotions are, is best "painted on" with an ordinary flat paint brush and allowed to dry. The addition of the local anaesthetic type of sedative (e.g. I per cent phenol) should be used with care and discrimination and not in any routine way for reasons I have already set out. Other useful lotions are:- - p per cent aluminium acetate, liq. plumbi subacet, I teaspoonful to a pint of tepid water or milk and water; I to 5 per cent ichthyol in water; $\frac{1}{2}$ to I per cent silver nitrate or tannic acid; potassium permanganate $I: 6,000$; normal saline or boracic lotion. On occasion these lotions may be applied as "tepid compresses" with several layers of gauze. They will then need frequent re-wetting and the layer of gauze next the skin should be left in position on each occasion so $\frac{0}{3}$ that the "inflamed epidermis" is not aggravated by undue friction.

In the papular phase itching is, as a rule, the $\frac{0}{6}$ most prominent symptom and special attention should be directed towards allaying it. Here again lotions are frequently the most effective, but $\overrightarrow{0}$ a more lasting effect may be produced by the use of $\frac{}{5}$ an emulsifying cream. There are several suitable $\overline{\frac{\omega}{p}}$ bases available but I have found the following $\stackrel{\widetilde{\alpha}}{\circ}$ formula provide a generally useful and elegant result:-

$\left.\begin{array}{c}\text { R } \\ \text { Lotio Calaminae B.P.C. }\end{array}\right\}$.. ää equal parts $\stackrel{\vec{\omega}}{\vec{\omega}}$

To this can be added such soothing ingredients $\frac{8}{8}$ as $\mathrm{I}-5$ per cent liq. picis carbonis, I-5 per cent 3 ichthyol or I per cent gentian violet.

In the papular phase, Pastes, such as the famous $\stackrel{N}{\sim}$ one bearing Lassar's name, are frequently most $\vec{f}$ effective. They have the advantage of being semiporous and combine well with such additions as $\omega$

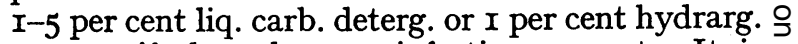
ammon if there be any infection present. It is usually better to use oil for their removal and it is $\frac{\vec{O}}{2}$ generally not desirable to make vigorous attempts to remove every trace of paste. In my opinion $\overrightarrow{0}$ pastes are usually best spread on to linen which $\frac{8}{8}$ of then applied to the affected region.

In the vesicular phase with accompanying weeping it is desirable to dry the discharges and prevent their becoming septic. Whilst this can often be done by "tepid compresses," of such lotions as boracic, normal saline or pot. permang., it is frequently much better done by the cold $\vec{\circ}$ Boro-starch poultice. In this the starch-jelly 3 (containing I dr. boric acid to $\mathrm{I} \frac{1}{2} \mathrm{oz}$. starch) is spread about an inch thick on butter-muslin or linen and applied direct to the part. It is better $\frac{0}{3}$ changed in about an hour or the starch will begin to "set." This poultice will also be found invaluable in "cleaning up" any crusted or scabbed surface.

In the lichenified or extremely dry and chronic phases a rather more stimulating treatment is 9 called for and here ointments are especially useful. $\frac{7}{5}$ Tar, resorcin and salicylic acid are the most generally useful ingredients and the following for- $\tilde{N}$ mula, for which I am indebted to Dr. J. M. H. MacLeod, has stood me in remarkably good stead:-

$\begin{array}{llll}\text { R } & \text { Liq. Picis Carb. } & \ldots & \text { m. } \\ \text { Zinc Oxide } & \ldots & \text { dr. } & \text { i } \\ \text { Paraff. Molle } & \ldots & \text { oz. } & \text { i }\end{array}$

Sometimes even more stimulating applications, such as $\frac{1}{2}-$ I per cent derobin or I-4 per cent chrysa- 
robin are needed but they should always be used with circumspection.

It is in these very chronic and resistant phases that suitably divided and administered exposures to X-rays are most useful. This form of treatment should always be carried out by someone conversant with the reactions of the skin to irradiation as well as versed in the technical details of the procedure.

\section{Washing and Baths}

About the advisability of washing and bathing there is great diversity of views. Many observers hold that water, especially if hard and combined with soap, is invariably harmful to eczema. Others think that it is advisable to keep lesions as clean as possible and so prevent sepsis. As ever the middle course between these two extremes is the wise one and each case should be judged on its merits. The use of normal saline or sodi bicarb. solution (a teaspoonful to a pint) seldom aggravates any but the most acute eczema, and many patients find the present substitutes for olive oil most irritating. I think it is far more important that, when an area is bathed or washed, the fluid used should be neither hot nor cold, but as near body temperature as feasible.

\section{Internal Treatment}

"There is no medicine which has a curative effect in eczema" (Sequeira). One has only to see the lengthening list of remedies advised from various sources to realise the truth of this. Of course, it is obvious that any derangement of other organs or systems of the body should be corrected. Much help will be given to the local measures by relieving chronic constipation or stasis, dyspepsia, alkalinising persistently acid urine, relieving nervous tension by suitable sedatives, but none of these are specifics. Much has been written of excellent results of oral or intravenous administration of calcium, sodium thiosulphate, a ro per cent strontium bromide in glucose solution (ekzebrol) but my experience has been disappointing. The same may be said of non-specific measures (e.g. auto-haemotherapy, aolan, sterile milk, etc.) and it is very difficult to exclude the psychological effect of convincing the patient that "something definite is being done." I have often thought that a well-placed kick would be just as effective as Io c.c. of whole blood in the buttock. I think we are all relieved that the days of arsenic as a universal remedy for eczema are past.

I would like to rail against the indiscriminate use of the sulphonamides internally in eczema. Beside the fact that there is no real indication for their use, there is the definite danger of their provoking a sensitivity and the risk of the even more lethal effect they may have on the blood forming and other systems.

\section{Diet}

I suppose eczema is the disease which suffers more than any other from the fads of theorists. Patients are given lists of what they may not indulge in, which no doubt they find most impressive, but for which there is little, if any, real reason, and it is seldom that two lists agree. The rules of diet for eczema should be largely those for the management of any form of illness. If the eruption is extensive and acute and the patient is confined to bed the diet should be bland and largely fluid. It is obviously undesirable to permit cutaneous vaso-dilators, such as tea, coffee, alcohol and condiments in any phase of eczema, but one must always consider how miserable one may easily make a patient by cutting off his accustomed solace of a comforting night cup. Generally speaking, I think one of our professional ancestors gave sound advice when he said "eat what you like and what you know agrees with you." I am in complete agreement with Sulzberger (I940) when he writes "except fore cases of infantile eczema (atopic) and perhaps in exceptional cases of atopic dermatitis in adults in which some specific food or foods are at fault, diet plays rather a small part in the treatment of eczematous dermatitis." Our experiences during the period of war and peace rationing will bear this out. In diet for eczema as for most things in life, the best advice is "moderation."

To sum up, the management of a case of eczema consists in:-

(I) Prolonged search for an external "sensitising" contact and, if found, elimination of it.

(2) Encouraging and allowing both the patient and the eruption to get well rather than trying to force them.

\section{REFERENCES}

(I) voN PIRQUET, Muenchner Medizinische Wochenschrift, r9o6, 30, I457. Translation by $M$. B. Sulzberger.

(2) BLOCH, BRUNO, The Role of Idiosyncrasy and Allergy in Dermatology I929, Arch. Derm. and Syph., 19, p. I75 et seq.

(3) SEQUEIRA, J. H., Diseases of the $S$ kin, 3rd. ed., p. 95 et seq.

(4) INGRAM, J. T., Eczema, Brit. Jour. Derm. and Syph., I935, pp. 47-64

and 502 .

(5) SULZBERGER, MARION B., Dermatologic Allergy, I940, p. 100 et

(6) SULŻBERGER, MARION B., ibid., p. 470.
(6) 